\title{
Estratégias Econômicas e Sociais para Anticoagulação de Pacientes com Fibrilação Atrial
}

\author{
Economy- and Social-Based Strategies for Anticoagulation of Patients with Atrial Fibrillation \\ Andressa Zulmira Avila Guerrero, ${ }^{1}{ }^{\circledR}$ Enia Lucia Coutinho, ${ }^{1}$ Marcos Bosi Ferraz, ${ }^{1}$ Claudio Cirenza, ${ }^{1}$ Marcelo \\ Cincotto Esteves dos Santos, ${ }^{1}$ José Roberto Ferraro, ${ }^{1}$ Angelo Amato Vincenzo de Paola ${ }^{1}$ \\ Universidade Federal de São Paulo, ${ }^{1}$ São Paulo, SP-Brasil
}

\section{Resumo}

Fundamento: A fibrilação atrial é um problema de saúde pública associado com um risco cinco vezes maior de acidente vascular cerebral e mortalidade. A análise de custos é importante para a introdução de novas terapias, e deve ser reconsiderada em situações especiais, tais como a pandemia do coronavírus em 2020.

Objetivo: Avaliar os custos (em um período de um ano) relacionados à terapia anticoagulante e a qualidade de vida de pacientes com fibrilação atrial tratados em um hospital público universitário.

Métodos: Os custos do paciente foram aqueles relacionados à anticoagulação e calculados pela média de custos mensais da varfarina ou de anticoagulantes orais diretos (DOACs). As despesas não médicas, como alimentação e transporte, foram calculadas a partir de dados obtidos de questionários. O questionário brasileiro SF-6D foi usado para medir a qualidade de vida. Valores $\mathrm{p}<0,05$ foram considerados estatisticamente significativos.

Resultados: A população do estudo consistiu em 90 pacientes, 45 em cada braço (varfarina vs. DOACs). Os custos foram 20\% mais altos no grupo dos DOACs (US\$55 532,62 vs. US\$46 385,88), e principalmente relacionados ao preço dos medicamentos (US\$23 497,16 vs. US\$1903,27). Os custos hospitalares foram mais altos no grupo da varfarina (US\$31 088,41 vs \$24 604,74), e relacionados às visitas ao ambulatório. Ainda, as despesas não médicas foram duas vezes maiores no grupo varfarina $(\$ 13394,20$ vs $\$ 7$ 430,72). A equivalência de preço entre os dois medicamentos seria alcançada por uma redução de $39 \%$ no preço dos DOACs. Não foram observadas diferenças quanto à qualidade de vida.

Conclusões: Os custos totais foram mais altos no grupo de pacientes tratados com DOACs que no grupo da varfarina. No entanto, uma redução de cerca de $\mathbf{4 0} \%$ no preço dos DOACs tornaria viável a incorporação desses medicamentos no sistema de saúde público brasileiro.

Palavras-chave: Custos de Cuidados de Saúde; Anticoagulantes; Varfarina; Fibrilação Atrial.

\begin{abstract}
Background: Atrial fibrillation is a public health problem associated with a fivefold increased risk of stroke or death. Analyzing costs is important when introducing new therapies and must be reconsidered in special situations, such as the novel coronavirus pandemic of 2020.

Objective: This study aimed to evaluate the costs related to anticoagulant therapy in a one-year period, and the quality of life of atrial fibrillation patients treated in a public university hospital.

Methods: Patient costs were those related to the anticoagulation and calculated by the average monthly costs of warfarin or direct oral anticoagulants (DOACs). Patient non-medical costs (eg., food and transportation) were calculated from data obtained by questionnaires. The Brazilian SF-6D was used to measure the quality of life. P-values $<0.05$ were considered statistically significant.

Results: The study population consisted of 90 patients, 45 in each arm (warfarin vs direct oral anticoagulants). Costs were 20\% higher in the DOAC group (\$55,532.62 vs \$46,385.88), and mainly related to drug price $(\$ 23,497.16$ vs $\$ 1,903.27)$. Hospital costs were higher in the warfarin group $(\$ 31,088.41$ vs $\$ 24,604.74)$ and related to outpatient visits. Additionally, non-medical costs were almost twice higher in the warfarin group (\$13,394.20 vs $\$ 7,430.72$ ). Equivalence of price between the two drugs could be achieved by a $39 \%$ reduction in the price of DOACs. There were no significant group differences regarding quality of life.

Conclusions: Total costs were higher in the group of patients taking DOACs than those taking warfarin. However, a nearly $40 \%$ reduction in the price of DOACs could make it feasible to incorporate these drugs into the Brazilian public health system.

Keywords: Health Care Costs; Anticoagulants; Warfarin; Atrial Fibrillation.
\end{abstract}

Full texts in English - http://www.arquivosonline.com.br

Correspondência: Angelo Amato Vincenzo de Paola •

Rua Napoleão de Barros, 715. CEP 04021-001, São Paulo, SP - Brasil

E-mail: depaola@uol.com.br

Artigo recebido em 19/08/2020, revisado em 21/01/2021, aceito em 24/02/2021

DOI: https://doi.org/10.36660/abc.20200921 


\section{Introdução}

A fibrilação atrial (FA) não valvar é um problema de saúde pública associado a um risco cinco vezes maior de acidente vascular cerebral (AVC) e mortalidade. ${ }^{1-4}$ A varfarina é amplamente utilizada nessa condição, ${ }^{5-7}$ e os pacientes geralmente necessitam de consultas ambulatoriais frequentes para se alcançar um tempo na faixa terapêutica (TTR, time in therapeutic range) adequado. ${ }^{8-11} \mathrm{O}$ controle laboratorial e as visitas ao ambulatório são não apenas uma carga econômica, como também uma questão de distanciamento social, ambos fatores importantes desde que a Organização Mundial da Saúde declarou a doença do coronavírus 2019 (COVID-19) como uma emergência de saúde pública internacional. Estudos sobre medidas preventivas e o controle efetivo de infecções durante a pandemia foram realizados. ${ }^{12,13}$

Os anticoagulantes orais diretos (DOACs), são um avanço importante na terapia de anticoagulação na FA, facilitaram o controle clínico, com boa eficácia e segurança. ${ }^{14-16}$ No cenário atual de pandemia, a terapia anticoagulante com DOACs é adequada uma vez que prescinde de visitas frequentes a serviços de saúde, favorecendo o distanciamento social. ${ }^{17}$ Contudo, os DOACs ainda não estão disponíveis no sistema de saúde no Brasil. Somente $30 \%$ dos pacientes no país possuem seguro saúde privado e acesso aos DOACs para prevenção de AVC. ${ }^{18}$

Recursos limitados dificultam a implementação de novas tecnologias, o que representa um problema na saúde pública. Avaliações da economia ajudam a aliviar a carga de recursos escassos, melhorando a eficácia alocativa do financiamento dos serviços de saúde. ${ }^{19,20}$

Este estudo teve como objetivo avaliar os custos relacionados à terapia anticoagulante (varfarina vs. DOACs) no manejo da FA, utilizando custos do mundo real em pacientes tratados em um hospital universitário pelo sistema público de saúde.

\section{Métodos}

\section{População do estudo}

Este foi um estudo observacional e retrospectivo. Calculouse um tamanho amostral de 89 pacientes com base no ambulatório de FA da Universidade Federal de São Paulo (680 pacientes), em que a prevalência de varfarina foi de $92 \%$ e a de DOACs de $8 \%$ somente. Um total de 90 pacientes foram recrutados. A amostragem foi realizada por conveniência para equilíbrio dos braços de tratamento (por exemplo, para cada paciente em uso de DOAC incluído, um paciente em uso de varfarina foi convidado a participar). O estudo foi aprovado pelo Comitê de Ética da Universidade Federal de São Paulo (\#79785717.2.0000.5505). Os pacientes que não compreenderam ou não aceitaram a assinar o termo de consentimento não foram incluídos.

Todos os pacientes foram incluídos em 2018 e os custos foram analisados no período de acompanhamento de 12 meses. A coleta de dados a partir dos prontuários médicos não afetou a frequência de visitas dos pacientes ao hospital ou a dosagem de varfarina ou DOACs, estabelecidas a critério da equipe de anticoagulação. Para a coleta de dados, desenvolvemos dois questionários utilizando o REDCAP (Research Electronic Data Capture), ${ }^{21}$ que consiste em uma aplicação web segura para a criação e gerenciamento online de pesquisas e banco de dados:

1. Questionário clínico/econômico: incluiu itens a respeito de dados demográficos (idade, sexo, profissão, educação), história clínica (comorbidades), e dados econômicos (salário, renda familiar, tipo de transporte, tempo de viagem).

2. Operacional: quantificou os custos individuais dos participantes na instituição. A soma dos valores foi feita por meio do Key Performance Indicators for Health (KPIH), um sistema de cálculo e análise de custos de nossa instituição.

\section{Custos}

O estudo comparou os custos de duas intervenções similares para identificar a de menor custo enquanto se mantinha um nível desejável de qualidade. Os custos foram classificados em duas categorias - custos dos pacientes e custos do hospital. ${ }^{22}$

\section{Custos do paciente}

Os custos médicos do paciente incluíram os custos relacionados à anticoagulação e foram calculados pelos custos mensais médios com varfarina ou DOACs pagos e identificados pelo paciente. Os custos não médicos dos pacientes foram calculados a partir de dados fornecidos no questionário, tais como os custos com refeições/bebidas, transporte, e outros gastos. Para o cálculo com transporte, foi considerado o meio de transporte utilizado por cada paciente no dia da visita ao hospital. Para os pacientes que necessitaram de transporte público, consideramos o preço da passagem/ticket de ônibus e o número de passagens/ tickets usados por dia. Para os pacientes que utilizaram carro próprio, considerou-se a distância entre a casa e a clínica de anticoagulação e o consumo de combustível.

Os custos foram calculados usando os preços de janeiro de 2020 e corrigidos de acordo com o índice de preços no consumidor, e convertidos a dólares americanos em 09 de abril de 2020 (1 dólar americano $=5,10$ reais). Neste estudo, todos os custos foram expressos em dólares americanos (US\$).

$\mathrm{O}$ custo de oportunidade, que se referiu à quantidade de renda que o paciente e/ou familiar deixou de ganhar no trabalho durante o estudo, foi incluído na análise de perda de produtividade. A perda de produtividade no trabalho foi calculada com base nos dias de absenteísmo e perda no salário.

\section{Custos hospitalares}

Os custos hospitalares referiram-se aos serviços oferecidos a cada paciente, e coletados por meio do sistema KPIH. Esse consiste em um indicador de desempenho e quantifica os custos diretos e indiretos de cada departamento. Os custos médicos diretos incluíram os relacionados com medicamentos, internações, sala de emergência, visitas ao ambulatório, exames laboratoriais e de imagem. Os custos indiretos 
incluíram os gastos gerais e administrativos (por exemplo, despesas com departamento de contabilidade e departamento pessoal), e despesas incorridas em áreas do hospital não geradoras de renda que devem ser alocadas sobre as áreas geradoras de renda do hospital.

\section{Qualidade de vida (SF-6D)}

Questionário de qualidade de vida de 36 itens (SF-36) é um questionário genérico de qualidade de vida composto de 36 itens/questões, distribuídos entre oito domínios, resumidos em um componente físico e um componente mental. Em 2002, Brazier et al. ${ }^{23}$ revisaram o SF-36 e desenvolveram um novo índex que estabeleceu uma nova classificação de status de saúde usando seis domínios (SF-6D). Uma versão brasileira do SF-6D possibilita gerar valores de utilidade, e escores mais altos indicam uma melhor qualidade de vida. O SF-6D descreve saúde em seis dimensões (função física, limitação nas funções, função social, dor, saúde mental, e vitalidade), de dois a seis níveis de gravidade, descrevendo, assim 18 mil estados de saúde possíveis. ${ }^{24}$

\section{Análise estatística}

Para análise estatística dos dados, as variáveis quantitativas foram expressas em média e desvio padrão (DP) e as variáveis qualitativas como valores absolutos e porcentagens. A normalidade da distribuição dos dados quantitativos foi avaliada pelo teste de Shapiro-Wilk. Para comparações entre os grupos, o teste $t$ de Student não pareado foi usado para dados quantitativos, e o teste do qui-quadrado para dados qualitativos. Valores $\mathrm{p}<0,05$ foram considerados estatisticamente significativos. Todas as análises foram realizadas pelo programa SPSS Statistics, versão 25.0 (IBM Corp., Armonk, NY, EUA).

\section{Resultados}

\section{A) Características sociodemográficas}

A população do estudo foi composta por 90 pacientes, 45 em cada braço do estudo (varfarina vs. DOACs; Tabela 1). Todos os participantes no braço de antagonistas da vitamina $\mathrm{K}$ (AVK) usavam varfarina. Os DOACs utilizados pelos pacientes foram distribuídos da seguinte maneira: rivaroxabana $70 \%$, apixabana $18 \%$, dabigatrana $10 \%$, e edoxabana $2 \%$. Os fatores de risco e condições basais estão descritos na Tabela 1. As médias do escore CHA2DS2-VASc foi três, e a média do escore HAS-BLED foi um.

\section{B) Custos hospitalares, custos pessoais, e custo total}

Os custos hospitalares foram maiores no grupo varfarina (Tabela 2); seis (12\%) pacientes do grupo varfarina e 26 $(52 \%)$ do grupo DOACs iam rotineiramente às consultas no ambulatório com um familiar acompanhante. Os custos relacionados às perdas de produtividade dos pacientes e acompanhantes estão apresentados na Tabela 3 e os custos totais na Tabela 4.

C) Visão geral dos custos baseada no Sistema Único de Saúde (SUS)

Os DOACs foram 10 vezes mais caros que a varfarina; no entanto, os custos totais do braço dos DOACs foram aproximadamente 1,2 vezes (20\%) maiores. A enorme diferença entre esses dois tipos de anticoagulantes (DOACs US\$23 497,16 vs. varfarina US\$1903,27) pode ser muito reduzida se considerarmos os demais custos envolvidos no estudo. Nesse cenário, os custos indiretos do paciente foram relacionados à perda de produtividade, e os custos diretos a transporte e alimentação, o que contribuíram ao aumento

Tabela 1 - Características sociodemográficas e fatores de risco nas condições basais dos pacientes com fibrilação atrial em uso de varfarina e anticoagulantes orais diretos (DOACs) atendidos no ambulatório da Universidade Federal de São Paulo em 2018

\begin{tabular}{lccc}
\hline & varfarina $(\mathbf{n}=\mathbf{4 5})$ & DOACs $(\mathbf{n}=\mathbf{4 5})$ & Valor $\mathbf{p}$ \\
\hline Idade (anos) & $68 \pm 9$ & $72 \pm 10,5$ & 0,07 \\
\hline Mulheres (\%) & $51 \%$ & $53 \%$ & 0,08 \\
\hline Ensino fundamental, $\mathrm{n}(\%)$ & $32(71)$ & $24(53)$ & 0,08 \\
\hline Ensino superior, $\mathrm{n}(\%)$ & $12(27)$ & $36(80)$ & 0,04 \\
\hline Aposentados, $\mathrm{n}(\%)$ & $34(76)$ & $379,83 \pm \$ 300,05$ & 0,06 \\
\hline Salário (US\$) & $304,03 \pm \$ 211,34$ & 0,12 \\
\hline Necessidade de transporte público, $\mathrm{n}(\%)$ & $37(84)$ & $23(52)$ & 0,01 \\
\hline Tempo de viagem por consulta (hr.: min.) & $2: 50 \pm 1: 24$ & $27(60)$ & 0,45 \\
\hline Hipertensão, $\mathrm{n}$ (\%) & $36(82)$ & $11(24)$ & 0,03 \\
\hline Diabetes mellitus, $\mathrm{n}$ (\%) & $14(31)$ & $8(17)$ & 0,48 \\
\hline Insuficiência cardíaca, $\mathrm{n}$ (\%) & $9(20)$ & $6(13)$ & 0,86 \\
\hline Infarto do miocárdio, $\mathrm{n}(\%)$ & $6(13)$ & $5(11)$ & 0,10 \\
\hline AVC/Ataque isquêmico transitório, $\mathrm{n}(\%)$ & $6(13)$ & 0,74 \\
\hline
\end{tabular}

As variáveis quantitativas são expressas em média \pm desvio padrão, e as variáveis qualitativas em valores absolutos e porcentagens. Ensino fundamental completo é definido como a conclusão de cinco anos dessa fase escolar. Valores $p<0,05$ foram considerados estatisticamente significativo. AVC: acidente vascular cerebral 


\begin{tabular}{lcccc}
\hline \multicolumn{1}{l}{ Tabela 2 - Custos da instituição por serviços prestados ao paciente } & & \\
\hline & No. & Custos com varfarina (US\$) & No. & Custos com DOACs (US\$) \\
\hline Exames laboratoriais & 1021 & 2347,17 & 671 & 1467,61 \\
\hline Exames de imagem & 208 & 3110,75 & 318 & 5976,94 \\
\hline Visitas ao hospital & 688 & 12778,02 & 348 & 4628,20 \\
\hline Sala de emergência & 71 & 9496,54 & 85 & 7475,8 \\
\hline Internações & 8 & 3355,93 & 10 & 5056,81 \\
\hline Total (US\$) & & 31088,41 & & 24604,74 \\
\hline
\end{tabular}

DOACs: anticoagulantes orais diretos.

Tabela 3 - Custos totais do paciente para recebimento do tratamento

\begin{tabular}{lccc}
\hline & & Custos com varfarina (US\$) (n=45) & Custos com DOACs (US\$) (n=45) \\
\hline Paciente/Cuidador & Transporte & 2790,32 & 1011,10 \\
\hline \multirow{2}{*}{ Perda de produtividade } & Alimentação & 2669,67 & 605,25 \\
& Medicamentos & 1903,27 & 23497,16 \\
\hline Total & Paciente & 7393,77 & 5185,43 \\
\hline
\end{tabular}

DOACs: anticoagulantes orais diretos.

Tabela 4 - Despesas totais (referente a 12 meses) dos pacientes com fibrilação atrial atendidos no ambulatório da Universidade Federal de São Paulo em 2018 em tratamento com varfarina $(n=45)$ ou anticoagulantes orais diretos $(n=45)$

\begin{tabular}{lcc} 
& Custos com varfarina (US\$) ( $\mathrm{n=45)}$ & Custos com DOACs (US\$) ( $\mathrm{n=45)}$ \\
\hline Custo pessoal & 15297,47 & 30 927,88 \\
\hline Custo hospitalar & 31088,41 & 24604,74 \\
\hline Total & 46385,88 & 55532,62 \\
\hline
\end{tabular}

DOACs: anticoagulantes orais diretos.

dos custos no braço da varfarina. A diferença de US\$9146,74 (Fórmula 1) poderia ser eliminada com uma redução de aproximadamente $40 \%$ (Fórmula 2) no preço dos DOACs.

Fórmula 1 - diferença real: diferença entre os custos totais com DOACs e varfarina:

DOACs total - varfarin total

US\$55 532,62 - US\$46 385,88

Diferença real US\$9146,74

Fórmula 2 - Redução necessária no preço dos DOACs para torná-la comparável ao preço da varfarina:

Preço dos ADOCs - Diferença real difference (fórmula 1) = US\$23 497,16 - \$9 146,74=\$14 350,42

(61\% do preço dos AODCs ou redução de 39\% no preço do medicamento)

\section{D) Questionário Short Form (SF-6D)}

O SF-6D foi usado para medir a qualidade de vida dos participantes (Tabela 5). Os escores de utilidade mais baixos foram relatados pelo domínio da função social, mostrando um importante déficit social em ambos os braços. A média do escore de utilidade do SF-6D foi 0,649 para o grupo AVK vs 0,641 para o grupo DOACs (Tabela 5). Não foi observada diferença em nenhum outro parâmetro de qualidade de vida entre os grupos.

\section{Discussão}

A ampla utilização da varfarina no Brasil é justificada por seu baixo custo e boa eficácia. No entanto, a necessidade de controle laboratorial e variações no TTR são causas frequentes de complicações clínicas e interrupção no tratamento medicamentoso, ${ }^{25,26}$ o que pode tornar o manejo do paciente ainda mais desafiador. ${ }^{27}$ A subutilização de anticoagulantes é mais pronunciada em países de renda média, como em países da América do Sul, em que o uso chega a ser inferior a 40\%. ${ }^{28}$

Os DOACs foram introduzidos no Brasil em 2012, ${ }^{29} \mathrm{com}$ uma clara vantagem sobre outras terapias..$^{30-32}$ Infelizmente, devido aos altos custos, os DOACs têm sido praticamente utilizados exclusivamente na prática privada. Contudo, a necessidade de testes laboratoriais e frequentes visitas ao hospital para o manejo da terapia com varfarina geram 
Tabela 5 - Média de escores dos domínios do questionário de qualidade de vida SF-6D dos pacientes com fibrilação atrial atendidos no ambulatório da Universidade Federal de São Paulo em 2018 em tratamento com varfarina $(n=45)$ ou anticoagulantes orais diretos (DOACs) ( $n=45)$

\begin{tabular}{lcccc}
\hline & varfarin & DP & DOACs & DP \\
\hline Função física & 0,692 & 0,338 & 0,633 & 0,429 \\
\hline Limitações nas funções & 0,564 & 0,356 & 0,570 & 0,364 \\
\hline Função social & 0,240 & 0,302 & 0,220 & 0,274 \\
\hline Dor & 0,877 & 0,324 & 0,906 & 0,393 \\
\hline Saúde mental & 0,680 & 0,424 & 0,802 & 0,322 \\
\hline Vitalidade & 0,446 & 0,236 & 0,448 & 0,220 \\
\hline
\end{tabular}

Os escores do SF-6D varia de 0 a 1, em uma escala onde 0 é igual ao pior estado de saúde e 1 significa o melhor estado de saúde. DOACs.: anticoagulantes orais diretos; DP: desvio padrão.

problemas logísticos que se tornaram mais críticos na pandemia da COVID-19 devido à necessidade de distanciamento social/

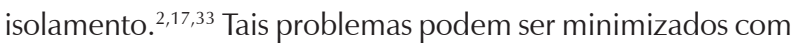
a incorporação dos DOACs ao SUS. Uma análise de custo efetividade dá evidência de que, apesar dos custos mais elevados, os DOACs são mais vantajosos, com melhor custo efetividade que a varfarina. ${ }^{28,34-39}$

A avaliação econômica com foco somente na assistência financeira direta não leva em consideração alguns fatores importantes para a análise global, como transporte, alimentação, absenteísmo no trabalho, e estresse. ${ }^{40,41} \mathrm{O}$ grupo da varfarina apresentou mais custos diretos (por exemplo, alimentação: US\$2669,67 vs. US\$605,25 para varfarina e DOACs, respectivamente; transporte: US\$2790,32 vs. US\$1011,10 para varfarina e DOACs, respectivamente), bem como um maior número de visitas e testes laboratoriais (varfarina US\$1709 vs. DOACs US\$1019). No entanto, nosso estudo mostrou que os custos totais do tratamento com DOACs (US\$55 532,62) foram mais altos que com varfarina (US\$46 385,88). Essa diferença no preço total foi diretamente relacionada ao preço dos DOACs (DOACs US\$23497,16 vs. varfarina US\$1903,27).

Os DOACs não são atualmente cobertos pelo SUS principalmente por seu elevado preço. Estudos locais com foco não somente em custos institucionais como também em custos sociais são necessários. O manejo da varfarina é difícil; muitos clínicos gerais são céticos em prescrever ou manter prescrições prévias de AVK. Nosso estudo reforça a ideia de que os DOACs podem ser fornecidos pelo serviço público de saúde. Silva et al. ${ }^{42}$ em um estudo sobre a qualidade do controle da anticoagulação em pacientes com FA não valvar, tratados com varfarina em um serviço de saúde privado, relataram que mais de $60 \%$ dos pacientes estavam abaixo do alvo desejado (em termos de TTR), o que se associou com maiores custos.

Com base nas nossas análises, o preço "ideal" dos DOACs seria US\$14 350,42 (fórmula 2). Nesta situação, o controle de anticoagulação com varfarina e DOACs teria custos comparáveis. Pacientes que não requerem monitoramento frequente estão mais propensos a praticar o distanciamento social, requerido pelas autoridades sanitárias durante a pandemia da COVID-19. ${ }^{40}$ Nesse contexto, seria muito desejável o desenvolvimento de uma abordagem viável de incorporação de DOACs nas políticas públicas de saúde, contribuindo para um sistema de saúde único e justo. Reduções nos custos no grupo DOACs são a única possibilidade de redução de custo nesse cenário. Uma estratégia simples para isso seria a redução do preço dos DOACs, calculado para esse propósito neste estudo.

O reembolso dos gastos no SUS é inadequado, com pouca transferência de pagamentos para a saúde pública. Assim, medidas administrativas são necessárias para promover um equilíbrio racional dos custos e acuidados acessíveis e conscientes. Sugerimos que algumas intervenções nos preços poderiam gerar um retorno econômico positivo, e a prestação eficiente de serviços de saúde, levando a um cenário viável para o uso de DOACs na prática clínica.

Interessante notar que um grande número de consultas não influenciou a qualidade de vida relacionada à saúde no grupo varfarina. Provavelmente, o alto grau de humanismo de nossa equipe de anticoagulação na FA tenha compensado a falta de apoio social e desconforto causados pelas visitas repetitivas ao hospital, conforme descrito em estudos realizados em outros países. ${ }^{43-45} \mathrm{O}$ fornecimento de DOACs a pacientes com FA é justificado e necessário não apenasdurante uma pandemia, como também em cenários clínicos e epidemiológicos normais.

\section{Limitações}

Nosso estudo apresentou algumas limitações. O estudo não foi randomizado, e desfechos como AVC, mortalidade e adesão não foram analisados. As despesas foram analisadas durante um período de 12 meses, e possivelmente um acompanhamento de longo prazo possa revelar mais benefícios.

\section{Conclusão}

Os custos no grupo DOACs mostraram-se maiores que no grupo varfarina. Custos não medicamentosos são importantes cargas no grupo varfarina, demandando estratégias econômicas em saúde racionais para o manejo da doença. Uma redução de $40 \%$ no preço das DOACs pode ser importante para a incorporação desses medicamentos no SUS, uma política viável durante a pandemia da COVID-19. 


\section{Agradecimentos}

O presente estudo foi realizado com apoio da Coordenação de Aperfeiçoamento de Pessoal de Nível Superior - Brasil (CAPES) - Código de Financiamento 001.

\section{Contribuição dos autores}

Concepção e desenho da pesquisa e Análise e interpretação dos dados: Guerrero AZA, Coutinho EL, Ferraz MB, Paola AAV; Obtenção de dados e Redação do manuscrito: Guerrero AZA, Coutinho EL, Paola AAV; Análise estatística: Guerrero AZA, Coutinho EL, Cirenza C, Paola AAV; Obtenção de financiamento: Paola AAV; Revisão crítica do manuscrito quanto ao conteúdo intelectual importante: Guerrero AZA,
Coutinho EL, Ferraz MB, Cirenza C, Santos MCE, Ferraro JR, Paola AAV.

\section{Potencial conflito de interesse}

Não há conflito com o presente artigo

\section{Fontes de financiamento}

O presente estudo foi financiado pela CAPES.

\section{Vinculação acadêmica}

Este artigo é parte de dissertação de mestrado de Andressa Zulmira Avila Guerrero pela Universidade Federal de São Paulo.

\section{Referências}

1. Kirchhof P, Benussi S, Kotecha D, Ahlsson A, Atar D, Casadei B, et al. 2016 ESC Guidelines for the Management of Atrial Fibrillation Developed in Collaboration with EACTS. Eur J Cardiothorac Surg. 2016;50(5):1-88. doi: 10.1093/ejcts/ezw313.

2. Guzik TJ, Mohiddin SA, Dimarco A, Patel V, Savvatis K, Marelli-Berg FM, etal. COVID-19 and the Cardiovascular System: Implications for Risk Assessment, Diagnosis, and Treatment Options. Cardiovasc Res. 2020;116(10):1666-87. doi: $10.1093 / \mathrm{cvr} / \mathrm{cvaa} 106$.

3. Lam A, Goulouti E, Roten L. The Search for Atrial Fibrillation and its Impact on Public Health. Swiss Med Wkly. 2017;147:14447. doi: 10.4414/ smw.2017.14447.

4. January CT, Wann LS, Alpert JS, Calkins H, Cigarroa JE, Cleveland JC Jr, et al. 2014 AHA/ACC/HRS Guideline for the Management of Patients with Atrial Fibrillation: Executive Summary: A Report of the American College of Cardiology/American Heart Association Task Force on Practice Guidelines and the Heart Rhythm Society. Circulation. 2014;130(23):2071-104. doi: 10.1161/CIR.0000000000000040.

5. Ge B, Zhang Z, Zuo Z. Updates on the Clinical Evidenced Herb-Warfarin Interactions. Evid Based Complement Alternat Med. 2014;2014:957362. doi: 10.1155/2014/957362.

6. Choi S, Oh DS, Jerng UM. A Systematic Review of the Pharmacokinetic and Pharmacodynamic Interactions of Herbal Medicine with Warfarin. PLoS One. 2017;12(8):0182794. doi: 10.1371/journal.pone.0182794.

7. Massaro AR, Lip GYH. Stroke Prevention in Atrial Fibrillation: Focus on Latin America. Arq Bras Cardiol. 2016;107(6):576-89. doi: 10.5935/ abc. 20160116

8. Wan Y, Heneghan C, Perera R, Roberts N, Hollowell J, Glasziou P, et al. Anticoagulation Control and Prediction of Adverse Events in Patients with Atrial Fibrillation: A Systematic Review. Circ Cardiovasc Qual Outcomes. 2008;1(2):84-91. doi: 10.1161/CIRCOU.

9. Lip GY, Lane DA. Stroke Prevention in Atrial Fibrillation: A Systematic Review. JAMA. 2015;313(19):1950-62. doi: 10.1001/jama.2015.4369.

10. Mohammed S, Aljundi AH, Kasem M, Alhashemi M, El-Menyar A. Anticoagulation Control Among Patients with Nonvalvular Atrial Fibrillation: A Single Tertiary Cardiac Center Experience. J Adv Pharm Technol Res. 2017;8(1):14-8. doi: 10.4103/2231-4040.197370.

11. Pinho-Costa L, Moreira S, Azevedo C, Azevedo P, Castro E, Sousa H, et al. APOLLO I: Anticoagulation Control in Atrial Fibrillation. Rev Port Cardiol. 2015;34(5):337-45. doi: 10.1016/j.repc.2014.11.008.

12. Ruan Q, Yang K, Wang W, Jiang L, Song J. Clinical Predictors of Mortality Due to COVID-19 Based on an Analysis of Data of 150 patients from Wuhan, China. Intensive Care Med. 2020;46(5):846-8. doi: 10.1007/s00134-020-05991-x.
13. Sohrabi C, Alsafi Z, O’Neill N, Khan M, Kerwan A, Al-Jabir A, et al. World Health Organization Declares Global Emergency: A Review of the 2019 Novel Coronavirus (COVID-19). Int J Surg. 2020;76:71-6. doi: 10.1016/j. ijsu.2020.02.034.

14. Caturano A, Galiero R, Pafundi PC. Atrial Fibrillation and Stroke. A Review on the Use of Vitamin K Antagonists and Novel Oral Anticoagulants. Medicina. 2019;55(10):617. doi: 10.3390/medicina55100617.

15. Hinojar R, Jiménez-Natcher JJ, Fernández-Golfín C, Zamorano JL. New Oral Anticoagulants: A Practical Guide for Physicians. Eur Heart J Cardiovasc Pharmacother. 2015;1(2):134-45. doi: 10.1093/ehjcvp/pvv002.

16. Hirschl M, Kundi M. Safety and Efficacy of direct Acting Oral Anticoagulants and Vitamin K Antagonists in nonvalvular Atrial Fibrillation - A Network Meta-Analysis of Real-World Data. Vasa. 2019;48(2):134-47. doi: 10.1024/0301-1526/a000746.

17. Wilder-Smith A, Freedman DO. Isolation, Quarantine, Social Distancing and Community Containment: Pivotal Role for Old-Style Public Health Measures in the Novel Coronavirus (2019-nCoV) Outbreak. J Travel Med. 2020;27(2):1-6. doi: 10.1093/jtm/taaa020.

18. Cantú-Brito C, Silva GS, Ameriso SF. Use of Guidelines for Reducing Stroke Risk in Patients With Nonvalvular Atrial Fibrillation: A Review From a Latin American Perspective. Clin Appl Thromb Hemost. 2018;24(1):22-32. doi: $10.1177 / 1076029617734309$.

19. Silva EN, Silva MT, Pereira MG. Health Economic Evaluation Studies: Definition and Applicability to Health Systems and Services. Epidemiol Serv Saude. 2016;25(1):205-7. doi: 10.5123/S1679-49742016000100023.

20. Ammerman AS, Farrelly MA, Cavallo DN, Ickes SB, Hoerger TJ. Health Economics in Public Health. Am J Prev Med. 2009;36(3):273-5. doi: 10.1016/j.amepre.2008

21. Harris PA, Taylor R, Thielke R, Payne J, Gonzalez N, Conde JG. Research Electronic Data Capture (REDCap)--a Metadata-Driven Methodology and Workflow Process for Providing Translational Research Informatics Support. J Biomed Inform. 2009;42(2):377-81. doi:

22. Vitez TS. Principles of Cost Analysis. J Clin Anesth. 1994;6(5):357-63. doi: 10.1016/s0952-8180(05)80003-0.

23. Brazier J, Roberts J, Deverill M. The Estimation of a Preference-Based Measure of Health from the SF-36. J Health Econ. 2002;21(2):271-92. doi: 10.1016/s0167-6296(01)00130-8.

24. Ciconelli RM, Ferraz MB, Kowalski S, Pinheiro GR, Sato El. Brazilian Urban Population Norms Derived from the Health-Related Quality of Life SF-6D. Qual Life Res. 2015;24(10):2559-64. doi: 10.1007/s11136-015-0991-x. 
25. Clarkesmith DE, Pattison HM, Khaing PH, Lane DA. Educational and Behavioural Interventions for Anticoagulant Therapy in Patients with Atrial Fibrillation. Cochrane Database Syst Rev. 2017;4(4):CD008600. doi: 10.1002/14651858.CD008600.pub3.

26. Ruff CT, Giugliano RP, Braunwald E, Hoffman EB, Deenadayalu N, Ezekowitz $M D$, et al. Comparison of the Efficacy and Safety of New Oral Anticoagulants with Warfarin in Patients with Atrial Fibrillation: A Meta-Analysis of Randomised Trials. Lancet. 2014;383.

27. Lakkireddy DR, Chung MK, Gopinathannair R, Patton KK, Gluckman TJ, Turagam M, et al. Guidance for Cardiac Electrophysiology during the COVID-19 Pandemic from the Heart Rhythm Society COVID-19 Task Force; Electrophysiology Section of the American College o.

28. Vinereanu D, Lopes RD, Bahit MC, Xavier D, Jiang J, Al-Khalidi HR, et al. A Multifaceted Intervention to Improve Treatment with Oral Anticoagulants in Atrial Fibrillation (IMPACT-AF): An International, Cluster-Randomised Trial. Lancet. 2017;390(10104):173.

29. Khoo CW, Lip GY. Insights from the Dabigatran Versus Warfarin in Patients with Atrial Fibrillation (RE-LY) Trial. Expert Opin Pharmacother. 2010;11(4):685-7. doi: 10.1517/14656560903530691.

30. Diener HC, Ntaios G, O'Donnell M, Easton JD. Non-Vitamin-K Oral Anticoagulants (NOACs) for the Prevention of Secondary Stroke. Expert Opin Pharmacother. 2018;19(14):1597-602. doi: 10.1080/14656566.2018.1515913.

31. Steffel J, Verhamme P, Potpara TS, Albaladejo P, Antz M, Desteghe L, et al. The 2018 European Heart Rhythm Association Practical Guide on the Use of Non-Vitamin K Antagonist Oral Anticoagulants in Patients with Atrial Fibrillation: Executive Summary. Euro.

32. Lip GYH, Keshishian A, Li X, Hamilton M, Masseria C, Gupta K, et al. Effectiveness and Safety of Oral Anticoagulants Among Nonvalvular Atrial Fibrillation Patients. Stroke. 2018;49(12):2933-44. doi: 10.1161/ STROKEAHA.118.020232.

33. Barnes GD, Burnett A, Allen A, Blumenstein M, Clark NP, Cuker A, et al. Thromboembolism and Anticoagulant Therapy during the COVID-19 Pandemic: Interim Clinical Guidance from the Anticoagulation Forum. J Thromb Thrombolysis. 2020;50(1):72-81. doi: 10.1007 .

34. Souza CPR, Santoni NB, Melo TG, Figueiredo MJO, Darrieux FCC, Piegas LS, et al. Cost-Effectiveness and Cost-Utility Analyses of Dabigatran Compared with Warfarin in Patients with Nonvalvular Atrial Fibrillation and Risk Factors for Stroke and Systemic Emb.
35. Marcolino MS, Polanczyk CA, Bovendorp AC, Marques NS, Silva LA, Turquia $\mathrm{CP}$, et al. Economic Evaluation of the New Oral Anticoagulants for the Prevention of Thromboembolic Events: A Cost-Minimization Analysis. Sao Paulo Med J. 2016;134(4):322-9. doi: 10.15.

36. Lamb LC, DiFiori M, Comey C, Feeney J. Cost Analysis of Direct Ora Anticoagulants Compared with Warfarin in Patients with Blunt Traumatic Intracranial Hemorrhages. Am Surg. 2018;84(6):1010-4.

37. Briere JB, Bowrin K, Wood R, Holbrook T, Roberts J. The Cost of Warfarin Treatment for Stroke Prevention in Patients with Non-Valvular Atrial Fibrillation in Mexico from a Collective Perspective. J Med Econ. 2017;20(3):266-72. doi: 10.1080/13696998.2016.1.

38. Costa J, Fiorentino F, Caldeira D, Inês M, Pereira CL, Pinheiro L, et al Cost-Effectiveness of Non-Vitamin K Antagonist Oral Anticoagulants for Atrial Fibrillation in Portugal. Rev Port Cardiol. 2015;34(12):723-37. doi: 10.1016/j.repc.2015.07.004.

39. Kamae I, Hashimoto Y, Koretsune Y, Tanahashi N, Murata T, Phatak H, et al. Cost-Effectiveness Analysis of Apixaban against Warfarin for Stroke Prevention in Patients with Nonvalvular Atrial Fibrillation in Japan. Clin Ther. 2015;37(12):2837-51. doi: 10.10

40. Vivas D, Roldán V, Esteve-Pastor MA, Roldán I, Tello-Montoliu A, Ruiz-Nodar JM, Recommendations on Antithrombotic Treatment during the COVID-19 Pandemic. Position Statement of the Working Group on Cardiovascular Thrombosis of the Spanish Society of Cardio.

41. Hermans C, Lambert C. Impact of the COVID-19 Pandemic on Therapeutic Choices in Thrombosis-Hemostasis. J Thromb Haemost. 2020;18(7):17945. doi: $10.1111 /$ jth. 14845 .

42. Silva PGMBE, Sznejder H, Vasconcellos R, Charles GM, Mendonca-Filho HTF, Mardekian J, et al. Anticoagulation Therapy in Patients with NonValvular Atrial Fibrillation in a Private Setting in Brazil: A Real-World Study. Arq Bras Cardiol. 2020;114(3):457-66.

43. Laäs DJ, Naidoo M. An Evaluation of Warfarin Use at an Urban DistrictLevel Hospital in KwaZulu-Natal Province, South Africa. S Afr Med J. 2018;108(12):1046-50. doi: 10.7196/SAMJ.2018.v108i12.13256.

44. Barnes GD, Acosta J, Graves C, Puroll E, Kline-Rogers E, Gu X, et al. Barriers to Integrating Direct Oral Anticoagulants into Anticoagulation Clinic Care: A Mixed-Methods Study. Res Pract Thromb Haemost. 2018;3(1):79-84. doi: 10.1002/rth2.12157.

45. Leminen A, Pyykönen M, Tynkkynen J, Tykkyläinen M, Laatikainen T. Modeling Patients' Time, Travel, and Monitoring Costs in Anticoagulation Management: Societal Savings Achievable with the Shift from Warfarin to Direct Oral Anticoagulants. BMC Health Serv. 